\title{
MODERNE-DEBATTEN AUF DER GRUNDLAGE DES KULTURELLEN TRANSFERS IM PESTER LLOYD DER 1900ER-JAHRE
}

\author{
ZSUZSA BOGNÁR \\ Lehrstuhl für Germanistik, Katholische Péter Pázmány Universität \\ zsbognar@btk.ppke.hu
}

\begin{abstract}
Die wichtigste deutschsprachige Tageszeitung Ungarns, der liberale Pester Lloyd (1854-1944) spielte in der Kulturvermittlung zwischen Ungarn und den anderen europäischen Ländern von Anfang an eine entscheidende Rolle. Um die Jahrhundertwende, als Literatur und Kunst den Erwartungen der institutionalisierten volksnationalen Ästhetik untergeordnet wurden, konnte seine Praxis des regelmäßigen Kulturtransfers in Form von kritischen und theoretischen Veröffentlichungen der Moderne zur Etablierung verhelfen. Der Beitrag stellt die einzelnen Etappen dieses Prozesses mit typischen Textbeispielen dar.
\end{abstract}

Schlüsselwörter: Moderne, Kulturtransfer, nationale Volkstümlichkeit, Ausgangs- und Zielkultur

\section{Theoretische und historische Vorüberlegungen}

Dem bekannten Kulturtransferforscher Wolfgang Schmale zufolge gibt es um die vorige Jahrhundertwende zwischen der Überschreitung der Grenzen von Nationalkulturen und den Modernisierungsprozessen einen klaren Zusammenhang: „Im großen und ganzen kann bezüglich des späten 19. Jahrhunderts von einem europäischen kulturellen System gesprochen werden, das Kultur im engeren Wortsinn, Politik und Wirtschaft umfasste, [...] und das seinen hervorragendsten Ausdruck in der Moderne um 1900 in den europäischen Metropolen fand."

Zur Etablierung der Moderne war es demnach unerlässlich, die im 19. Jahrhundert von Herder entworfenen Begriffe der nationalen Literatur und Kultur neu zu denken, und beide als Veränderungen unterworfene, heterogene oder hybride Gebilde zu akzeptieren. Interkulturelle Begegnungen führen zur Infragestellung homogenisierender Kulturkonzepte, seien sie durch die politische Ideologie der erwachenden Nationalstaaten forciert, oder sieht man sie, wie es in der Mehrheit der Länder der Österreich-Ungarischen Monarchie der Fall war, als Garanten für die Bewahrung der nationalen Selbstständigkeit an. 
Gleichzeitig hat Kulturtransfer - wie widersprüchlich das auch erscheinen mag - bei der Festigung der kulturellen Identität eine bedeutende Rolle. Für diese stellte Michel Espagne fest, dass kulturelle Wechselwirkungen - gegen die Erwartungen - nicht zur Verhinderung der Entfaltung und Aufbewahrung kultureller Identität führen, sondern dass sie im Gegenteil für ihre Entwicklung und Verstärkung förderlich sein können. ${ }^{2}$ Indem im Weiteren die kulturelle Strategie der ungarischen Moderne in ihrer Widerspiegelung in der deutschsprachigen Tageszeitung Pester Lloyd untersucht wird, werden beide obigen Konzepte der Kulturtransferforschung einbezogen und bestätigt.

Nach der Jahrhundertwende war das geistige Leben in Ungarn einander entgegengesetzten Einflüssen ausgesetzt. Zunächst als Folge der Niederschlagung des Freiheitskrieges gegen Österreich 1848/49, später, nach der Entstehung der Österreich-Ungarischen Monarchie 1867, verstärkte sich - als Reaktion auf die Rivalität der verschiedenen Nationalitäten innerhalb des Vielvölkerstaates - der Anspruch auf eine selbstständige Nationalliteratur. Andererseits hatte ein großer Teil der Intelligenz - zum Beispiel auf Grund ihrer Auslandsstudien - erkannt, dass die Modernisierung in Ungarn ohne Beachtung der europäischen Vorbilder nicht ausführbar sei. Dieses Dilemma spaltete das kulturelle Leben: Während die Vertreter der konservativen akademischen Institutionen die ästhetische Ideologie der sogenannten nationalen Volkstümlichkeit bevorzugten und ausschließlich volkstümliche oder historische Themen und Formen als echte Repräsentanten der nationalen Kultur akzeptierten, suchte die Mehrheit der jungen Künstler in den westeuropäischen Metropolen nach Vorbildern. Der wichtigste Bereich des Kulturkampfes war die Presse: nicht zufällig markiert in der ungarischen Literaturgeschichtsschreibung das Starten der Zeitschrift Nyugat 1908 die Entstehung der modernen Literatur, wobei allein der Titel 'Westen' auf die Orientierung der neuen Generation hindeutete. Dies weniger im Sinne der Anlehnung an die westeuropäische Literatur als vielmehr eines Bruchs mit der konservativen volksnationalen Kunsttradition. Nyugat hatte freilich ihre Vorgänger, aber ein ausgeprägtes Moderne-Bewusstsein, bzw. wiederkehrende Reflexionen auf die Moderne lassen sich in der damaligen Publizistik erst ab ihrem Erscheinen verfolgen. Dies berücksichtigend, werden in die Darstellung der Moderne-Debatte durch den Pester Lloyd solche Texte einbezogen, die in der Zeit um 1908, jedoch nicht später als 1914 entstanden sind.

\section{Das kulturelle Profil des Pester Lloyd nach der Jahrhundertwende}

Der Pester Lloyd galt als das wichtigste halboffizielle Zeitungsorgan der ungarischen Regierung, sein Zielpublikum war das gebildete Großbürgertum der Haupstadt. Das Blatt verpflichtete sich zur Verbreitung der liberalen Ökonomie 
und der ungarischen Kultur, zudem vertrat es die ungarischen politischen Interessen im Ausland. Da die hinter ihm stehende finanzielle Basis viel größer war als jene hinter den ungarischsprachigen Tageszeitungen, konnte der Pester Lloyd in Ungarn in der größten Auflagenhöhe erscheinen - und dies in einem Umfang von circa 20 Seiten, an Wochenenden sogar mit doppeltem Umfang. Durch die Deutschsprachigkeit des Pester Lloyd war der Charakter der kulturellen Rubriken in hohem Maße vorbestimmt, d.h. die Zeitung zeichnete sich von vornherein durch eine gewisse transnationale Einstellung aus; Um die Jahrhundertwende hatte der kulturelle Transfer im Feuilletonteil bereits eine Tradition von 50 Jahren. ${ }^{3}$

Unter dem Blickwinkel der Kulturtransferforschung verfügte der Pester Lloyd im Gegensatz zu den anderen, ungarischsprachigen Tageszeitungen noch über den Vorteil, eigene ausländische Berichterstatter zu beschäftigen. Sie berichteten regelmäßig über Theater, Kunst und sonstige kulturelle Ereignisse in Wien und Berlin, seltener Paris und Mailand.

Für die Literatur und Kultur war der Feuilletonteil zuständig, und bis etwa 1907 konnte hier die Moderne fast ausschließlich nur durch die Wiener Theaterberichte von Ludwig Hevesi Platz finden. Hevesi machte die Zeitungsleser in der Reihenfolge seiner Artikel mit den Stücken von Maeterlinck, Gabriele D'Annunzio, Oskar Wilde und Hofmannsthal bekannt, außerdem widmete er seinem Favoriten, G. B. Shaw, ein eigenes Feuilleton. ${ }^{4}$ Nach Hevesis Tod 1910 wurde die zeitgenössische ausländische Literatur durch Felix Salten referiert, aber auch durch andere wie etwa Julius Bab, um nur den bedeutendsten Kritiker aus dieser Reihe hervorzuheben. Der führende Hauskritiker, Max Rothauser (1863-1913), selbst ein mittelmäßiger Bühnenautor, vertrat dagegen eine konservative, moralästhetische Einstellung, er entrüstete sich z.B. bei einem Gastspiel der Berliner in Budapest sehr über Wedekinds Erdgeist als einem Exempel der Moderne:

[...] und dann fragt man sich: ob das die neue Literatur, die neue Kunst sei, um derentwillen es verlohne alldas zu vergessen, was uns bisher lieb und ehrwürdig gewesen?! Nein, und hundertmal Nein! Das ist überhaupt keine Literatur und die Kunst, die solche Schauer- und Jammerdramatik aufgreift, weil sie nichts Besseres findet, ist fürwahr zu bedauern. ${ }^{5}$

Ein solch emotionaler Ausbruch war allerdings für den Theaterkritiker Rothauser nicht typisch; man kann ihm als Verdienst anrechnen, dass er einer neuen Generation von Kritikern den Weg nicht versperrte. Ein großer Wandel ist nämlich ab 1907 mit dem Auftreten junger Kritiker und Essayisten wie Ernst Goth, ${ }^{6}$ Michael Josef Eisler, ${ }^{7}$ Georg Lukács und Ignotus zu verzeichnen. Sie alle waren damals jünger als 30 Jahre, alle hatten ein Universitätstudium zumindest angefangen, und die Genannten wurden regelmäßige Mitarbeiter der Zeitschrift Nyu- 
gat. Sie taten im Pester Lloyd auch ihr Möglichstes, um die Sache der modernen ungarischen Literatur voranzubringen. Ihre Feuilletons beschränkten sich nicht auf die gewohnte kritische Präsentierung von Neuerscheinungen; man findet bei ihnen auch theoretische Ansätze, die für die Notwendigkeit einer Erneuerung in Ungarn plädieren. Eine auffallende Gemeinsamkeit ist dabei die Überschreitung der Grenzen der nationalen Literatur: Als eine Selbstverständlichkeit wurde von ihnen angenommen, dass die literarische Erneuerung erst auf der Grundlage des kulturellen Transfers vollzogen werden konnte, wobei diese als paradigmatischer Fall für die Möglichkeit einer allgemeinen, kulturellen, gesellschaftlichen und ideologisch-politischen Modernisierung angesehen wurde. Aus den verschiedenen Aufsätzen - Kritiken und Essays - lässt sich quasi ein Modernisierungsprogramm auf der Grundlage des kulturellen Transfers zusammenstellen, dessen einzelne Punkte im Weiteren durch entsprechende Zitate aus den verschiedenen kulturreflexiven Texten - Rezensionen, Kritiken, Debatten - belegt werden.

\section{Exkurs über den Kulturnationalismus der Kisfaludy-Gesellschaft}

Anfang 1907 veröffentlichte der Pester Lloyd - allerdings nicht in dem meistgelesenen Feuilletonteil des Morgenblattes, sondern in einem aus zwei Seiten bestehenden, der letzten Aktualität dienenden Abendblatt - die Eröffnungrede der Jahresversammlung der stockkonservativen Kisfaludy-Gesellschaft, was bei dieser liberal gesinnten Zeitung als sehr ungewöhnlich galt. Der Präsident der Gesellschaft, Zsolt Beöthy (1848-1922), war Professor an der Budapester Universität und beharrte unter dem Einfluss des Positivismus auf dem Prinzip der Nationalcharakterologie. Dem Gedanken des literarischen Nationalismus verpflichtet, nannte er die Pflege der nationalen Charakteristika das höchste Ziel der Kisfaludy-Gesellschaft, die im öffentlichen kulturellen Leben ein hohes Ansehen genoss.

In seiner Eröffungsrede fokussierte Beöthy auf die Problematik, wie die ungarische Literatur und Kultur mit den immer größer werdenden Einflüssen des Auslandes umgehen solle. Als Vertreter der Wissenschaft wusste er, dass man sich den Wirkungen der Außenwelt nicht völlig verschließen kann und darf, gleichzeitig war er von der identitätsstiftenden Kraft der ,nationalen Seele” überzeugt. Dieser eignete er die besondere Fähigkeit zu, ,,alle fremde Wirkung in nationalem Geiste und zu nationalem Zwecke umgestalten" zu können:

Gleichwie die italienische Renaissance in Balassa und Zrinyi zu einer ungarischen Renaissance wurde, so dienten der Kräftigung und Entwicklung unserer nationalen Seele: Horatius in Virágh und Berzsenyi, Shakespeare in Katona, die deutsche Romantik in Karl Kisfaludy und dessen Schule, die englische in Jósika, 
das französische Vaudeville in den Volksstücken Szigligetis und Eduard Tóths. ${ }^{8}$

Die Inanspruchnahme solcher und ähnlicher literaturhistorischer Beispiele für eine eigenwillige Interpretation von Transfererscheinungen kommt auch in den derzeitigen Moderne-Debatten oft vor. Versteht man nämlich Kulturtransfer in dem Sinne, wie er von den Kulturhistorikern Michel Espagne und Michael Werner in den 1980er-Jahren herausgearbeitet wurde, als „ein[en] aktiv[en], durch verschiedene Mittlergruppen betriebene[n] Aneignungsprozess, der von den Bedürfnissen der Aufnahmekultur gesteuert wird", 9 dann fällt bei Beöthy die völlige Ignorierung kontingenter Vorteile der Ausgangskulturen auf. Er nennt keinen einzigen Vorteil, der für die ungarische als Zielkultur erstrebenswert wäre, sondern weist nachdrücklich einzig und allein darauf hin, dass die ungarischen Dichter bzw. literarischen Bewegungen immer wieder fähig waren, fremdkulturelle Einflüsse zu bestimmten Zwecken zu nutzen. Er übersieht den Kultivierungsbedarf der Ungarn; Kulturtransfer müsse sich ihm zufolge in „der Kräftigung und Entwicklung unserer nationalen Seele" erschöpfen.

Im Folgenden kommt er zum eigentlichen Anliegen seiner Eröffnungsrede und nennt jene Faktoren, die seiner Ansicht nach die nationalkulturellen Eigenheiten der ungarischen Literatur in Gefahr bringen: es sind die weltweit anerkanntesten ausländischen Autoren, die "Strömungen” und das soziale Thema, wobei übrigens diese drei Faktoren auch geeignet sind, die epochenspezifischen Merkmale der Moderne abzugeben:

Auf dieser geschichtlichen Grundlage taucht in ihrer wahren Bedeutung, und zwar nicht nur in ästhetischer, sondern geradezu in national-psychologischer Bedeutung die Frage auf, wie wir in dieser Beziehung Zola, Ibsen, Tolstoi und den Strömungen der sogenannten Modernen, dem Einflüsse der sozialen Ideen auf unsere Dichtung gegenüberstehen? Lebt und wirkt die vorhin erwähnte umgestaltende, assimilierende ungarische Fähigkeit und Tendenz in ihrer einstigen Kraft? Diese Frage weckt mit gutem Grund die Aufmerksamkeit, die Sorge und die Arbeit unser Aller, denen es am Herzen liegt, daß unsere Literatur auf dem Pfade der nationalen Entwicklung verbleibe, und in deren Kreisen auch weiterhin, entsprechend unserer nationalen Seele und den in uns wurzelnden Überlieferungen, ihre hehre Ausgabe erfülle. ${ }^{10}$

Allerdings muss man zugeben, dass die Volkstümlichkeit als eine Erwartung Beöthys in der Rede nicht erwähnt wurde; diese hätte eine thematische und formale Beschränkung nach sich gezogen, was mit den wissenschaftlichen Ansprü- 
chen eines Gelehrten kaum vereinbar gewesen wäre. ${ }^{11}$ Aus heutiger Sicht lässt sich Beöthy als Wissenschaftler mit essentialistischer Kulturauffassung einstufen.

\section{Auseinandersetzung mit der Ideologie und Ästhetik der nationalen Volkstümlichkeit}

Die der Moderne verpflichteten ungarischen Kritiker des Pester Lloyd fingen Ende 1908 an, energisch gegen die Nivellierung von Normen der nationalen Volkstümlichkeit aufzutreten. Zeitgenössische ausländische Autoren und Werke waren von ihnen auch schon davor in der Zeitung vorgestellt worden: 1907 besprach Ernst Goth Neuerscheinungen von Hermann Bahr und Alfred Kerr, Ignotus veröffentlichte einen geistreichen Artikel über Oskar Wilde, und Georg Lukács rezensierte den Essayband von Rudolf Kassner. ${ }^{12}$ Diese Artikel wiesen schon in die Perspektive der Moderne, sie enthielten aber noch keine Polemik und auch keinen Bezug zur ungarischen Literatur. Es fehlte also die explizite Berücksichtigung der Zielkultur, und auch von den offiziellen Erwartungen der nationalen Volkstümlichkeit wurde dabei keine Abgrenzung vorgenommen. Ein Beispiel für diese kritische Position stellt folgende Einleitung zu einem Artikel Ernst Goths dar:

Die Zeit der behäbigen Onkelhaftigkeit in der Kritik, des
wohlwollenden oder stirnrunzelnden Zensurausteilens von
anno Sarcey ist glücklicherweise ebenso vorbei, wie der fixen,
stets paraten Maßstäbe, des unentwegt entschiedenen Ja-und-
Neinsagens - dieses ganz lächerlichen Papsttums überhaupt. [...]
Doch einen klaren, festumschriebenen Charakter nach der Art
der früheren Zeitläufe hat die moderne Kritik auch heute nicht.
Ganz wie bei der Kunst des Theaters - des neuen, zeitgemäßen,
versteht sich - sind auch bei der Kritik Formen und Inhalte noch
in langsamem Werden. ${ }^{13}$

Nach dem Auftreten von Nyugat vermehrten sich die Artikel, die auf die verpönten Erwartungen reflektierten und sich für die Anerkennung der modernen ungarischen Literatur einsetzten. Indem der hauptberufliche Psychiater Michael Josef Eisler die „Jungungarische Novellistik” vorstellte, konnte man bei ihm sowohl auf das lange vermisste Moderne-Bewusstsein als auch auf eine ironische Ablehnung der volksnationalen Topoi treffen:

In den Hainen der ungarischen Poesie regen sich neue Geister. Allem Anscheine nach ist eine tote Zwischenzeit gründlich überwunden und hoffnungsvolle Talente bereiten eine repräsentative Zukunft vor. [...] 
Die hier antretende Reihe der Künstler [...] arbeitet unter den vorwurfsvollen Blicken gewisser Literaturethiker, die die Poesie der Csikósmutwilligkeit und des beschaulichen Bauerntums in ihnen vermissen. Daß sich inzwischen eine veritable ungarische Hauptstadt entwickelt hat mit dem Konglomerat unterschiedlicher und oft zweideutiger Existenzen, wollen sie keinem glauben. Indes sind aber schon viele Augen eröffnet worden, dank der Treffsicherheit einiger jungen Literaten. ${ }^{14}$

Der um diese Zeit berühmteste ,junge Literat" war zweifellos Endre Ady. Ernst Goth zeichnete 1909 über diese emblematische Figur der modenen Lyrik ein ausgezeichnetes Porträt und fühlte sich dabei auch genötigt, auf die Unübersehbarkeit der technischen Entwicklung hinzuweisen, die neue Lebensweisen und Kommunikatonsformen ermöglicht und eine Neudefinierung der obsolet gewordenen ästhetischen Ideologie der offiziellen Instanzen einfordert:

\begin{abstract}
Auch der Begriff nationaler Volkstümlichkeit ist Wandlungen unterworfen. Und das Nationalempfinden Adys, des Sohnes einer friedlichen, kulturbeflissenen, von Weltproblemen erfüllten Zeit, in der Eilzugsverbindungen und Telegraphenleitungen auch den ungarischen Gesichtskreis unendlich weiteten, kann nicht mehr dasselbe sein, wie jene Petőfis, der nie über die Grenze gekommen war und den Säbel nie abgeschnallt hatte. Adys Ungartum ist nur ungleich differenzierter, ungleich intellektueller, doch um nichts schwächer. Es ist das Ungartum eines aus höhergearteten westlichen Kulturen halb unwillig, aber einem unzähmbaren Drange folgend immer wieder Heimkehrenden $[\ldots]^{15}$
\end{abstract}

Wenn der zuletzt zitierte Satz darauf hinausläuft, dass selbst bei Ady, der durch seine Paris-Erfahrungen ein moderner Dichter wurde, die Heimatliebe unerschütterlich sei, wird übrigens die in der Einleitung betonte Erkenntnis der Kulturtransferforschung bestätigt, laut der kulturelle Begegnungen auf die Identität sogar eine stabilisierende Wirkung ausüben.

\title{
4. 1. Widerlegung des Vorwurfs des Kosmopolitismus
}

In der Weihnachtsnummer 1908 des Pester Lloyd erschien ein programmatischer Aufsatz von Ignotus unter dem Titel Alte und Junge. Ein Versäumnis der ungarischen Literaturgeschichtsschreibung ist, dass der Text bis heute nicht wahrgenommen wurde; er stellt nämlich eine Fortsetzung und Vertiefung eines anderen, hingegen bekannten Ignotus-Aufsatzes dar, der im selben Jahr in der letzten Num- 
mer von Nyugat unter dem Titel Kriegerische Vorbereitungen erschienen war. ${ }^{16}$ Dieser war eine Art von Antwort auf den gleich betitelten Artikel von Kálmán Mikszáth, einem der besten Prosaisten der ungarischen Literatur überhaupt, der allerdings 1908 bereits zur älteren Generation zählte. ${ }^{17}$ Damit ist Ignotus' Pester Lloyd-Veröffentlichung nicht als eine gelegentliche Meinungsäußerung über die Kontroversen zwischen konservativen und jungen Autoren anzusehen, sondern soll in diese Moderne-Debatte eingebunden werden. Es ist hier nicht der Ort, um auf die Einzelheiten des Wortduells einzugehen, aber es soll nicht unerwähnt bleiben, dass die ungarischsprachige Debatte auf der Seite von Ignotus' nicht frei von persönlichen Beleidigungen war. Wahrscheinlich deshalb versuchte er im Pester Lloyd eine objektive, argumentative Darstellung des Konfliktes. Vom eigenen Lager wurden seine Ausführungen positiv aufgenommen: Goth hat in seinem oben besprochenen Ady-Aufsatz auf sie zurückverwiesen, als er beteuerte, dass sich „hinter den Forderungen des poetischen Chauvinismus stets verkappte Rückständigkeit und die Unfähigkeit verbirgt, Entwicklungen zu sehen und zu begreifen". ${ }^{18}$

Ignotus zufolge bestehe der Konflikt zwischen 'Alten' und 'Neuen' in der Auffassung und Beurteilung des nationalen Wesens der Literatur. Der größte Vorwurf gegen die Moderne sei der „des unnationalen Wesens”, ein Phänomen, das im Weiteren als „Kosmopolitismus” bezeichnet wird. Die Forderung nach nationaler Ursprünglichkeit - eine Losung der nationalen Volkstümlichkeit - sei unhaltbar, weil in der ungarischen Volkskunst viele ethnisch-kulturell 'fremde' Einflüsse verschmolzen sind. Gegen den Vorwurf des Kosmopolitismus verteidigt er sich mit einem 'postkolonialen' Argument, wenn er sich auf die 'hybride' Beschaffenheit der ungarischen Kulturgeschichte beruft:

So wie die heutige Kalotaßeger Stickerei einst die Handarbeit der Siebenbürgischen Hofdamen war und wohl auf italienische Muster zurückgeführt werden kann, ist auch vieles andere, was heute als volkstümliche Spezialität angesehen wird, weder volkstümlich noch Spezialität. Die ungarische Volksmusik ist gregorianischen Ursprungs; das ungarische Metrum gleicht dem Metrum der Edda; die ungarischen Jahrmarktsbücher, aus denen sich die Phantasie des Volkes seit Jahrhunderten nährt, sind ihrer Herkunft nach die Arbeiten Boccaccios, Petrarcas, Enea Silvios; und wenn deren ungarischen Bearbeitern Gáspár Heltai, Gábor Pesti, Albert Szenci-Molnár das fremde Gewand in ihrem nationalen Charakter nicht schadete, warum sollte dem heutigen ungarischen Dichter in dieser Hinsicht die Einwirkung jener schaden, die seinem Gefühl, seiner Moral, seiner Intelligenz näher stehen und deren Einfluß die Nation nicht in die Krypta der Vergangenheit sperren, sondern sie unter die Teilhaber am lebendigen Leben der Gegenwart heben würde? ${ }^{19}$ 
Auch Ignotus zählt Fälle des kulturellen Transfers auf, wie es Beöthy getan hat, tut es aber mit einer anderen Akzentuierung. In seinen Beispielen vollzieht sich nicht eine restlose Assimilierung fremdkultureller Einflüsse, sondern wird eine Bereicherung des Eigenen durch sie anerkannt. Deshalb kann er im nächsten Schritt die ältere Generation - Josef Kiss, Emil Ábrányi, Eugen Rákosi, Ludwig Dóczy u.a. - herbeizitieren, bei der solche Aneignungsprozesse schon abgelaufen sind, und „die seither durchwegs zu nationaler Dichtkunst avanciert ist". ${ }^{20}$ Die Anklage gegen die moderne ungarische Literatur, dass sie ihre kulturelle Identität verleugne oder sogar aufzugeben beabsichtige, sei demnach sinnlos:

[...] wenn man dem ungarischen Dichter empfiehlt, konservativ
zu sein, da er sonst seinen nationalen Charakter verliere, so
bedeutet das im Grunde nichts anderes, als die Forderung, daß
er bei der Nachahmung Heines, Tennysons, Bérangers, Byrons,
Victor Hugos und Herweghs bleibe und nicht wage, etwa
Swinburne, Oskar Wilde, Baudelaire, Verlaine und Henri de
Régnier auf sich wirken zu lassen. ${ }^{21}$

Ignotus legt mit dieser Gegenüberstellung von Autoren, die verschiedenen kulturhistorischen Strömungen repräsentieren, nahe, dass es in dem Konkurrenzkampf zwischen den Alten und Jungen nicht um die Rivalisierung von Eigenem/ Nationalem und Fremdem/Kosmopolitischem geht, sondern um die Dominanz von Weltvorstellungen, bzw. diesen adäquaten künstlerischen Ausdrucksweisen, weshalb eine nationalkulturelle Festlegung der beiden Grupperungen irrelevant sei.

\subsection{Annahme der Position einer Zielkultur}

Goths Ady-Porträt zielt ebenfalls auf eine Apologie der modernen ungarischen Lyrik im Allgemeinen und des Dichters im Besonderen, wobei dessen Werk als „der poetische Ausdruck der nach Ungarn verpflanzten, an europäischen Kulturen gesättigten modernen Seele“ angesehen wird. ${ }^{22}$ Andererseits heißt es über Ady, es ginge es bei ihm nicht um die Sehnsucht ,nach geistig wertvoller Ungebung, sondern nur instinktmäßig [...] nach dem Rausch der Fremde, der wunderbar erregenden Mystik des Unbekannten“. ${ }^{23}$ Die beiden Feststellungen mögen insgesamt widersprüchlich wirken, und als Grund dafür kann man annehmen, dass es auch Goth notwendig schien, Ady von der Anklage des Kosmopolitismus freizusprechen. Deshalb verfolgte er die Taktik, den Dichter als ,einen im Westen heimischen Urmagyaren" darzustellen; gleichzeitig versäumte er aber nicht anzumerken, dass „Ady oftmals auch das Wort nicht rein begriffsmäßig, sondern 
musikalisch, als Stimmungsbehelf verwendet, wie Verlaine, Baudelaire, Mallarmé es taten". ${ }^{24}$

Indem es darum ging, den Stellenwert des Kulturtransfers für die ungarische Moderne zu bestimmen, zögerte Goth vor einer eindeutigen Festlegung. Ignotus zählte einfach historische Beispiele auf und unternahm dabei keine Differenzierung oder eine genauere Erklärung der Art und Weise der Beeinflussungen. Ludwig Hatvany ging einen Schritt weiter: In seinem Essay Das alte Ungarn legte er nahe, die europäische Literatur habe als Vorbild für die beiden Nationalgrößen des 19. Jahrhunderts, Petöfi und Arany, gegolten, und ihr eifriges Studium durch Arany habe zu dessen dichterischer Entwicklung wesentlich beigetragen.

Die konservative Literaturwissenschaft stufte Petőfi und Arany ausschließlich als volkstümliche Dichter ein und schätzte bei ihnen das Aufgreifen der nationalen Problematik und die stilistische Nähe der ungarischen Volkspoesie - bei Hatvany hingegen werden auch sie mit europäischen Maßstäben gemessen. Über Petőfi stellt dieser nämlich zusammenfassend fest: „Petöfi war eben der erste Dichter Ungarns von europäischer Bedeutung." ${ }^{25}$ Arany bewundert Hatvany besonders, weil dieser fähig war, sich „weit von allem intellektuellen Verkehr” zum „Kulturmenschen” heranzubilden: „Doch der Notar des weltverschlagenen Ortes überwindet die größten Schwierigkeiten, um Bücher zu beschaffen. Er liest Homer und Goethe, Dante und Shakespeare. »Ich esse Ilias, ich trinke die Odyssee. $"{ }^{26}$

Hatvanys literaturgeschichtlicher Essay wurde im Laufe der Zeit in vier verschiedenen Publikationsorganen in jeweils unterschiedlicher Länge veröffentlicht, zweimal deutsch und zweimal ungarisch. Drei Textvarianten erschienen unmittelbar nacheinander im Jahre 1910 - zunächst auf etwa den halben Umfang gestrafft im Pester Lloyd, danach in der gesamten Fassung in Nyugat und schließlich in der Neuen Rundschau, die vierte wurde - allerdings in einer stark reduzierten Version - erst nach Hatvanys Tod, in den Sammelband Könyvek és emberek aufgenommen. ${ }^{27}$ Durch den Vorabdruck im Pester Lloyd wird schon klar, dass Hatvany in seiner Darstellung der ungarischen Kultur stets das deutsche Publikum im Blick hatte, da er in derart großer Häufigkeit auf ausländische Parallelerscheinungen Bezug nimmt. Beim Vergleichen aller drei Textvarianten hat man trotzdem nicht den Eindruck, dass sich Hatvany durch diesen Essay bei der deutschsprachigen Kulturszene hätte einschmeicheln wollen - er war ja darauf gar nicht angewiesen. Indem er jede Inspiration, jede neue Entwicklungsstufe auf ausländische - westliche - Einflüsse zurückführt, kann man unter Berücksichtigung seiner Bestrebungen zur Vermittlung der ungarischen Kultur ins Ausland vermuten, dass er sowohl historisch als auch perspektivisch beweisen wollte, dass für die Modernisierung Ungarns die Beachtung ausländischer Vorbilder unerlässlich sei. 


\subsection{Forderung nach den zeitgenössischen europäischen Maßstäben in der Kunst}

Dass die moderne ungarische Literatur der Zeit angemessene ästhetische Maßstäbe brauche, und diese nicht von konservativen Institutionen wie der Kisfaludy Gesellschaft und der Akademie bestimmt werden können, wird von Eisler in der oben behandelten Rezension mit kategorischer Eindeutigkeit formuliert: „Nicht bloß die Noblesse, auch die 'Literatur verpflichtet'. Nur mit der Bekanntschaft des europäischen Niveaus darf sich heute ein Künstlertum hervorwagen und die Kritik muß denselben Gesichtspunkt wahren." 28 Es bedeutete damals ganz konkret, dass europäischen Maßstäbe auch bei der offiziellen Förderung ungarischer Autoren berücksichtigt werden sollten, der Medienerfolg junger ungarischer Talente im Ausland verdiene institutionelle Anerkennung und Würdigung.

Den Anlass zu dieser Forderung gab ein Skandal wegen der Verteilung der von Privatpersonen gestifteten akademischen Preise, wobei der Pester Lloyd dieser Debatte eine breite Medienöffentlichkeit verlieh. 1911 hatte Ludwig Hatvany in einem Feuilleton unter dem Titel Akademie und Bühne die Entscheidung der Akademie bei der Zuteilung des Vojnits-Preises für das beste dramatische Werk des Jahres heftig kritisiert. Er behauptete, die Institution sei geneigt, „das begrenzte Können der Lokalgrößen” anzuerkennen, „und sträubt sich nun gegen die international-bühnensichere Gewandtheit der Moderne". ${ }^{29}$ Damit meinte er die damaligen internationalen Kassenerfolge von Menyhért Lengyel (Taifun), Sándor Bródy (Die Amme, Die Lehrerin) und Franz Molnár (Der Teufel). Hatvany weist dabei nicht allein auf die reine Tatsache einer sehr positiven ausländischen Rezeption hin, sondern entdeckt auch die Medienöffentlichkeit als einen nicht zu umgehenden Beeinflussungsfaktor:

Wäre die Akademie in naher Beziehung zum Leben, würden nicht die drei Philologen: Heinrich, Négyesy und Ferenczy, über Theater urteilen, sondern Theaterschriftsteller säßen in der Jury, so müssten sie wissen, daß heute Theater- und Zeitungswesen in engstem Zusammenhang sind. Rostand und Maeterlinck, Reinhardt und Strauß, alle brauchen Zeitungen. Wo eine übermächtige Zeitung sich gegen junge Schriftsteller auflehnt, da erstarrt das Theaterwesen. Siehe: Wien. ${ }^{30}$

In der nächsten Nummer des Pester Lloyd folgte die Antwort - von keinem Geringeren als dem Generalsekretär der Akademie, Gustav Heinrich ${ }^{31}$ selbst. Sein Antwortbrief zeigt keine deklarative Rhetorik wie jene von Beöthy und er ist auch nicht frei von Ironie, sogar Selbstironie, wenn er über die Verantwortung redet, die der Akademie bei der Vergabe der Stiftungspreise zukommt. Die Vor- 
würfe Hatvanys in Bezug auf deren Beurteilungspraxis lehnt er jedoch mit einer paternalistischen Geste ab, indem er sich auf die üblichen Anständigkeitskonventionen beruft:

Nicht die Stoffe stoßen uns ab; wir wissen sehr gut, dass es keinen
Stoff gibt, den der Dichter nicht in Gold verwandeln könnte; aber
die Art der Behandlung, die Tendenz, die Huldigung vor dem
Pöbel, die Verirrungen des Geschmacks, von denen diese Stücke
wimmeln, das ist es, was uns weh tut [...] Es gab eine Zeit, wo
junge Mädchen und anständige Frauen nicht ins Orpheum gehen
konnten; heute können sie nicht ins Theater gehen oder - $-{ }^{32}$

\section{Hoffnung auf eine Selbstpositionierung als Ausgangskultur}

Zu guter Letzt sei erwähnt, dass die ungarische Moderne - wenn auch nur ganz sporadisch - die Ambition zeigte, im Kulturtransfer nicht ewig als Zielkultur eine Rezipierende zu sein, sondern - den skandinavischen Autoren vergleichbar - sich auch als Ausgangskultur positionieren zu können. Den damals aktuellen Hintergrund zu diesem letzten Punkt lieferte Ernst Lorsy ${ }^{33}$ mit seinem Bericht über die Veröffentlichung mehrerer ungarischer Prosabände in Deutschland um 1914. Bemerkenswert ist dabei, dass sich der Feuilletonist feststellt, dass ungarische Literatur endlich auch in Deutschland gelesen werde, allerdings ist er nicht vom Grund des Interesses beim deutschen Publikum angetan, denn:

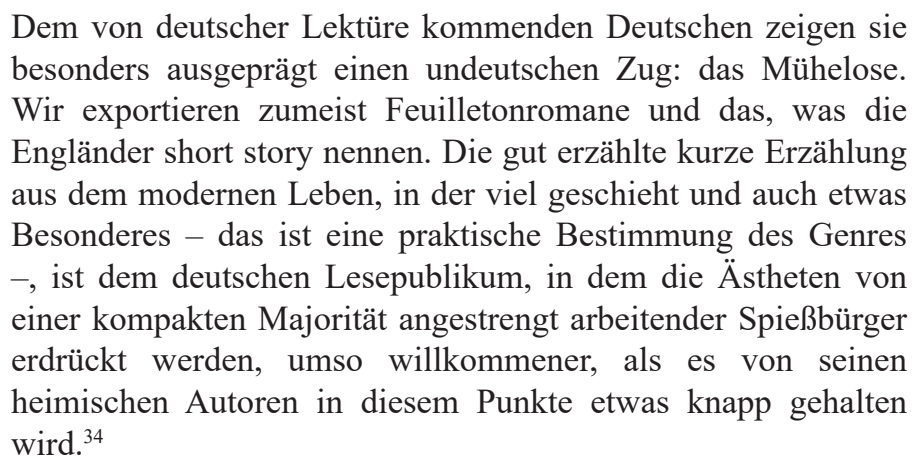

Lorsy reicht das alles nicht: Der Erfolg ungarischer Autoren beim breiten Publikum allein konnte ihn nicht zufriedenstellen:

Werden wir nie im Triumph kommen, Sieger über die Herzen, die sich uns festlich öffnen? Wie die Russen, wie die Nordländer? 
Wird sich uns das ex oriente lux nicht endlich einmal bewahrheiten wollen? Werden wir immer nur unterhalten, wenn's hochgeht, fesseln? Werden wir nie befruchten? Ein Blick aus der Vogelperspektive holt sich als Antwort ein zögerndes Vielleicht. ${ }^{35}$

Abschließend soll betont werden, dass eine solche konsequente Propaganda für die Moderne auf der Grundlage des Kulturtransfers in einer anderen Tageszeitung Ungarns um die Jahrhundertwende wohl kaum vertretbar gewesen wäre. Die renommierteste Kulturzeitschrift Nyugat veröffentlichte zwar Beispiele ausländischer Belletristik in reicher Anzahl, aber eine theoretisch-kritische Reflexion auf Kulturtransfererscheinungen hielt sie nicht für ihre Aufgabe. Polemiken rund um die Moderne waren damals jedenfalls ein fester Bestandteil der Publizistik.

\title{
Anmerkungen
}

\author{
Abkürzungen: \\ PL - Pester Lloyd \\ M. - Morgenblatt \\ A. - Abendblatt
}

1 Schmale, Wolfgang: Geschichte und Zukunft der Europäischen Identität. Stuttgart: Kohlhammer GmbH 2008, S. 145.

2 Espagne, Michel: Kulturtransfer und Fachgeschichte der Geisteswissenschaften. In: Comparativ 10 (2000), S. 42-61.

3 Ein quantitativer Vergleich mit der Neuen Freien Presse in den Stichjahren 1880, 1890, 1900 und 1910 bestätigte, dass für die literaturkritische Ausrichtung des Pester Lloyd eine ,internationale Vernetzung" charakteristisch war. Vgl: Melischek, Gabriele: Krisenkommunikation am Vorabend des Ersten Weltkriegs. In: Szendi, Zoltán (Hrsg.): Medialisierung des Zerfalls der Doppelmonarchie in deutschsprachigen Regionalperiodika zwischen 1880 und 1914. Wien: Lit Verlag 2014, S. 13-24, hier: S. 23.

4 Hevesi, Ludwig: Pelleas und Melisande - Gastspiel der Wiener Sezessionsbühne. PL, 22. Juli M. 175. 1900, S. 2.; Ders: Ein tragischer Tag. - Björnson's , Über unsere Kraft”. - D'Annunzio. - Eleonora Duse. -. PL, 6. April M. 84. 1902, S. 2-3. Ders.: Wiener Bühnen-Novitäten. (,,Salome”, von Oskar Wilde. - „Nachtmar”, von Erich Korn.) PL, 15. Dezember M. 301. 1903, S. 5.; Ders.: Bernard Shaw. PL, 1. März M. 52. 1903, S. 5.; Ders.: Wiener Theater. „Elektra”, von Hugo von Hofmannsthal. PL, 16. Mai M. 123. 1905, S. 1-2.

5 Rothauser, Max: ,,Der Erdgeist” (Eine Tragödie von Frank Wedekind. Dargestellt vom Ensemble des Berliner Neuen und Kleinen Theaters am Lustspieltheater zu Budapest.). PL, 7. Juni M. 137. 1904, S. 2.

6 Ernst Goth (um 1879-1942), urspr. Ernő Großmann, Journalist des Pester Lloyd ab 1906, Kritiker und Übersetzer. Nach dem Ersten Weltkrieg ließ er sich in Berlin nieder, 1923 zog er nach Florenz. In Deutschland versuchte er die ungarische Literatur, in erster Linie Endre Ady, populär zu machen.

7 Michael Josef Eisler (1882-1944) war Arzt, Psychoanalytiker, Essayist, Kritiker und Dichter. Er publizierte ab 1906 im Pester Lloyd, ab den 1920er-Jahren auch in psychoanalytischen 
Fachzeitschriften. Selbstständige Werke: Aubrey Beardsley egyénisége és müvészete. Budapest 1907, Elfenbeinturm. Sonette. Berlin 1910, Unerlöste Welt. Gedanken und Sprüche. Budapest 1935. Vgl. noch: Bognár, Zsuzsa: Michael Josef Eisler - Eine Textauswahl. Budapest-Piliscsaba 2002.

8 Beöthy, Zsolt: Literarische Strömungen. - Eröffnungsrede in der gestrigen Jahresversammlung der Kisfaludy-Gesellschaft. PL, 11. Februar A. 34. 1907, S. 1-2.

9 Middel, Matthias. Kulturtransfer,transferts culturels. http://docupedia.de/zg/middell_kulturtransfer_v1_de_2016 DOI: http://dx.doi.org/10.14765/zzf.dok.2.702.v1 (letzter Zugriff am 24. 04. 2019)

10 Beöthy 1907 (siehe Anm. 8).

11 Vgl. Németh G., Béla: Irodalomkritikai gondolkodás a pozitivizmus korában. Budapest: Akadémiai Kiadó 1981, S. 373-385.

12 Goth, Ernst: Zwei Kritiker. PL, 10. Februar M. 36. 1907, S. 24-25., Ignotus: Oskar Wilde. PL, 31. März M. 78. 1907, S. 17; Lukács, Georg: Motive. Essays von Rudolf Kassner. 15. Dezember M. 297. 1907, S. 14.

13 Goth 1907, S. 24 (siehe Anm. 8).

14 Eisler, Michael Josef: Jungungarische Novellistik. PL, 29. November M. 286. 1908, S. 19-20.

15 Goth, Ernst: Endre Ady. PL, 7. Februar M. 32. 1909, S. 22-23.

16 Ignotus: Hadi készülödések. Nyugat, Nr. 24. 1908, S. 449-454. Die Debatte setzte sich in Nyugat auch 1909 fort, siehe z. B. den gleich betitelten Artikel von Fenyő Miksa in der ersten Nummer 1909, S. 49-51.

17 Mikszáth, Kálmán: Hadi készülödések. Újság, 10. Dezember 1908.

18 Goth 1909, S. 22, (siehe Anm. 15).

19 Ignotus: Alte und Junge. PL, 25. Dezember M. 38., 1908, S. 3-5, hier: S. 4.

20 Ebd.

21 Ebd.

22 Goth 1909, S. 22 (siehe Anm. 15).

23 Ebd.

24 Ebd.

25 Hatvany, Ludwig: Das alte Ungarn. PL, 27. Februar M. 49. 1910, S. 25.

26 Ebd.

27 Die erwähnten weiteren Fassungen: Hatvany, Lajos: A magyar irodalom irodalom a külföld elött. Nyugat, 5 (1910), S. 273-293; Ders.: Das alte und das neue Ungarn. Neue Rundschau, 1910. S. 383-400; Ders.: A magyar irodalom a külföld elött. In: Könyvek és emberek. Budapest 1971. Vgl. noch: Bognár, Zsuzsa: Die ungarische Literatur vor dem Ausland, dargestellt von Ludwig Hatvany. In: Interkulturelle Erkundungen. Teil I. Hg. u.a. von Andrea Benedek, Renata Alice Crişan, Szabolcs János-Szatmári. Frankfurt am Main - Berlin - Bern: Peter Lang, S. 166-176.

28 Eisler 1908, S.19, (siehe Anm. 14).

29 Hatvany, Lajos: Akademie und Bühne. PL, 1. Februar M. 27. 1911, S. 1.

30 Ebd.

31 Gustav Heinrich (1845-1922) war positivistischer Literaturhistoriker, ab 1873 Professor für Germanistik an der Budapester Universität, zwischen 1905 und 1920 Generalsekretär der Ungarischen Akademie der Wissenschaften.

32 Heinrich, Gustav: Akademie und Bühne. PL, 2. Februar M. 28. 1911, S. 2.

33 Ernst Lorsy (1889-1961) war Publizist und Übersetzer. Für den Pester Lloyd arbeitete er ab 1913. Nach 1919 emigrierte er nach Wien, später nach Berlin. Nach Hitlers Machtübernahme wurde er Sekretär von Mihály Károlyi in Paris. Er starb in New York.

34 Lorsy, Ernst: Ungarische Literatur in Deutschland. 26. Februar M. 49. 1914, S. 1-2, hier: S. 1.

35 Ebd., hier S. 2. 
Open Access. This is an open-access article distributed under the terms of the Creative Commons Attribution 4.0 International License (https://creativecommons.org/licenses/by/4.0), which permits unrestricted use, distribution, and reproduction in any medium, provided the original author and source are credited, a link to the CC License is provided, and changes - if any - are indicated. (SID_1) 
